\title{
Electron Spin Resonance Spectrum of a New Anthraquinone Aglycone (Nodosin)
}

\author{
B. N. Misra, S. K. Gupta, and S. D. Sharma \\ Physics Department, Allahabad University, Allahabad, India \\ (Z. Naturforsch. 27 b, 1360-1362 [1972] ; received May 24, 1972)
}

Nodosin, free radical, Cassia nodosa, anthraquinone, ESR

\begin{abstract}
The Electron Spin Resonance study of 1,3,5,8-tetrahydroxy 2,7-dimethyl anthraquinone known as Nodosin has been carried out at room temperature and at X-band. The compound gives a norrow ESR line. The measurements of lineshape, linewidth and $g$-value have been made. A theoretical computation of dipolar width as well as narrowing due to exchange has been carried out with the help of Киво and TомiтA theory and finally a value for $J$, the exchange coupling parameter has been estimated. On the basis of these measurements Nodosin has been proposed to adopt an intermediate structure.
\end{abstract}

The earlier ESR studies ${ }^{1,2}$ on biological problems has shown that different parts of plants contain free radicals in them. Since then a large amount of work has been done in this direction ${ }^{3}$. In the present paper the ESR study of 1,3,5,8 tetrahydroxy 2-7 dimethyl anthraquinone named as Nodosin, obtained from chemical investigation ${ }^{4}$ of the flowers of Cassia nodosa ${ }^{5,6}$, has been undertaken. The study of lineshape, linewidth and $g$ value has been carried out. The KUBO and TOMITA theory of ESR linewidth has been utilized to obtain the narrowing due to exchange. The static magnetic susceptibility has also been studied. On the basis of these measurements a suitable intermediate structure has been proposed.

\section{Experimental Procedure}

Rizvi et al. ${ }^{4}$ after a chemical investigation of the flowers of Cassia nodosa could isolate a new anthraquinone glycoside named as Nodososide which after carrying out the acid hydrolysis etc. gave $D$ glucose and an Aglycone named as Nodosin with the molecular formula $\mathrm{C}_{16} \mathrm{H}_{12} \mathrm{O}_{6} \cdot \mathrm{C}_{2} \mathrm{H}_{5} \mathrm{OH}$ and the structural formula as given below

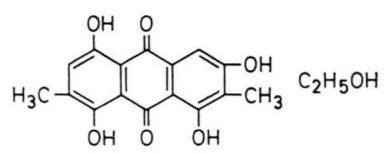

It contains a molecule of $\mathrm{C}_{2} \mathrm{H}_{5} \mathrm{OH}$ similar to water of crystallization and the same could not be removed from it even after a very long heating of the compound. The solid sample of Nodosin in powder form has been taken for ESR study. The B r u ke r X-band B-ER 402 refection type ESR spectrometer has been used for this study. The Hewlett Packard frequency converter as well as counter has been used for frequency measurements. The static magnetic susceptibility of the compound has been measured with the help of F a r a d a y method. The ESR spectrum of the powder sample has been recorded at room temperature.

\section{Results and Discussion}

\section{a) Magnetic susceptibility measurements}

The measured value of static magnetic susceptibility after applying the diamagnetic correction is

$$
\chi_{0}=0.74 \times 10^{-6} \text { per } g \text { in cgs units. }
$$

The value of $\left(\chi_{0}\right)_{\text {theor. }}$ assuming that each molecule has got one unpaired electron, has been obtained as

$$
\left(\chi_{0}\right)_{\text {theor. }}=3.52 \times 10^{-6} \text { per } \mathrm{g} \text { in cgs units. }
$$

The percentage radical concentration of the compound Nodosin using these two values of $\chi_{0}$ is found to be 21.3.

\section{b) ESR Measurement}

The ESR spectrum of Nodosin powder sample at room temperature has given a single line as shown in Fig. 1. The measurements of line shape, $g$ value and linewidth has been made from this record.

In order to have a better interpretation of the ESR data, line shape determination has first been carried out. The points corresponding to the value of $I(\mathrm{H}) / I_{\max }$ and $\left(H_{0}-H\right) / \Delta H_{\mathrm{pp}}$ taken from the experimental record have been plotted on the normalized theoretical Lorentzian and Gaussian 


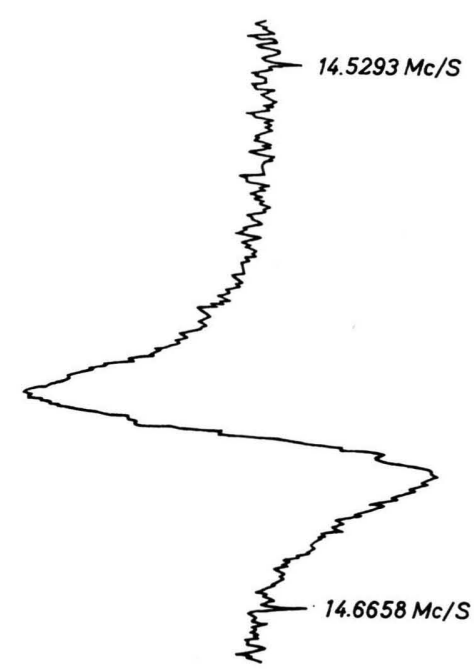

Fig. 1. E.S.R. of Nodosin.

lineshape graphs drawn with the help of the expressions given by POOLE ${ }^{7}$ as shown in Fig. 2 and it appears that the observed record is of L or e $\mathrm{n} \mathrm{z}$ ian shape. It has been further confirmed by obtaining the higher ratio of moments ${ }^{9}$. In general the actual shape of a line depends on the crystal structure,

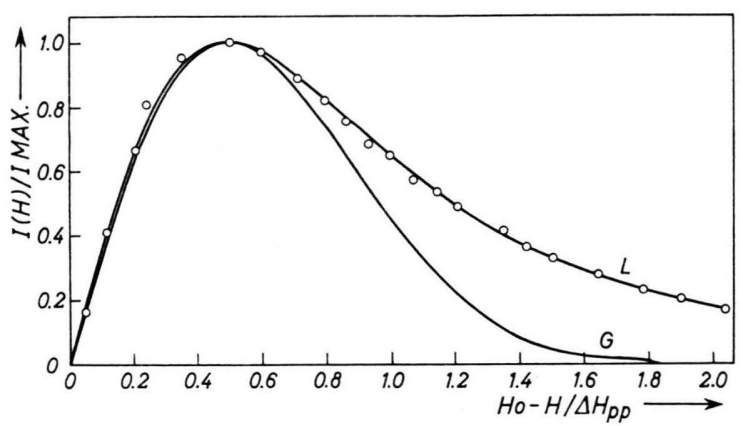

Fig. 2. Plot of calculated values of $I(\mathrm{H}) / I_{\max }$ versus $\left(\mathrm{H}_{0}-\mathrm{H}\right) /$ $\Delta \mathrm{H}_{\mathrm{pp}}$ along with the observed values in the case of Nodosin. Theoretical Lorentzian and Gaussian lineshapes. $\bigcirc \circ \circ$ Observed points for Nodosin.

magnetic dilution and exchange coupling. In ESR, the main cause of Lorentzian lineshape is the presence of exchange narrowing 8,9 . The presence of Lorentzian shape of line simply suggests that the compound has large exchange narrowing and behaves similar to that of DPPH and other solid free radicals cited in the literature ${ }^{3}$.

The peak to peak linewidths have been measured from different records and their mean value with their mean standard deviation is equal to $4.11 \pm 0.05$ oersteds. The modified expression ${ }^{10}$ for linewidth based on Kubo and Tomita theory ${ }^{11}$ has been used here. The expressions for different parameters are as:

Half width between half powerpoints

Dipolar width

$$
\Delta \omega \text { in radian } / \mathrm{sec}=4.18 \omega_{10}^{2} / \omega_{20}
$$

$$
\omega_{10}^{2} \text { in } \operatorname{radian}^{2} / \mathrm{sec}^{2}
$$

$$
=3.79 \mathrm{~g}^{4} \beta^{4} \hbar^{-2} \mathrm{~d}^{-6}
$$

Narrowing due to exchange $\quad \omega_{20}$ in $\mathrm{rad} / \mathrm{sec}$

$$
=3.65|J| / \hbar
$$

Exchange integral $|\boldsymbol{J}|$ in Joules $=\hbar \omega_{20} / 3.65$

Since the crystal structure of the compound is not known hence in order to determine the value of mean spin distance $d$ as a very rough approximation, it is assumed that it has a simple cubic structure then

where

$$
\sum_{k} r_{\mathrm{jk}}^{-6}=8.4 \mathrm{~d}^{-6}
$$

Using the values of molecular weight $M=346$, density $\varrho=0.95$ and $N$, the Avogadro number, $r_{\mathrm{jk}}$ $=8.5 \AA$ has been found out. The dipolar width $\omega_{10}^{2}$, narrowing due to exchange $\omega_{20}$, exchange integral $J$ obtained with the help of expressions given above are as follows:

$$
\begin{aligned}
& \omega_{10}^{2}=7.9 \times 10^{17} \mathrm{radian}^{2} \mathrm{sec}^{-2} \\
& \omega_{20}=53.1 \times 10^{9} \mathrm{radian} \mathrm{sec}^{-1} \\
& |\boldsymbol{J}|=1.5 \times 10^{-24} \text { Joules }
\end{aligned}
$$

A high value of dipolar width $\omega_{10}^{2}$ is obtained but it is sufficiently modified by the exchange and with the result that a narrow ESR line has been obtained. This simply shows that the conclusions drawn from the ratio of moments and lineshape determination that the system should have large exchange narrowing is confirmed here obtaining a high value of $\omega_{20}$ and $J$.

The $g$ value has been measured from the experimental record by usual method. The mean value of $g$ is equal to $2.0037 \pm 0.0001$. The value of $g$ found greater than 2.0023 here simply indicates that the electron is not free from orbital effect.

The entire data obtained from ESR measurements show that the compound Nodosin behaves like an organic free radical and so it should contain an unpaired electron. The present structure of the compound does not approve its free radical behaviour. Hence it can be concluded that the compound probably adopts an intermediate structure either by 


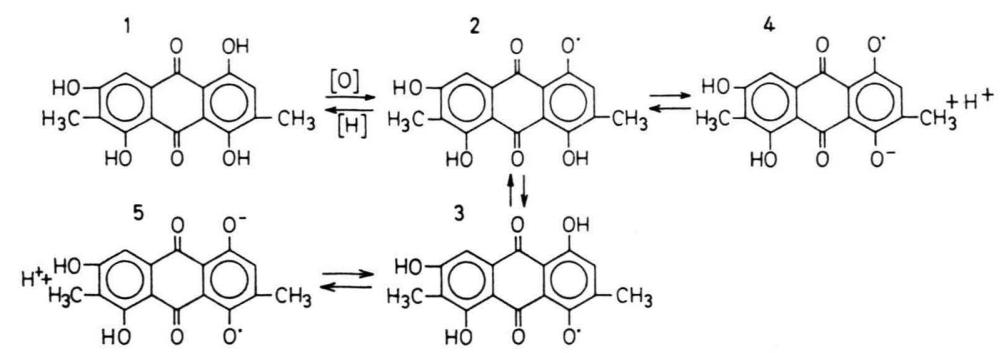

1 1,3,5,8-tetrahydroxy-2,7-dimethylanthraquinone (Nodosin)

atmospheric oxidation or reduction which may be represented as

Nodosin on account of this transformation ${ }^{12,13}$ to a semiquinone behaves as a free radical and gives a single sharp ESR line.

1 B. Commoner, J. Townsend, and G. E. Pake, Nature [London] 174, 689 [1954].

2 P. B. Sogo, N. G. Pon, and M. Calvin, Proc. nat. Acad. Sci. USA 42, 710 [1956].

3 D. J. E. Ingram : Free Radical as Studied by ESR, Butterworth Scientific Publication 1958.

4 S. A. I. Rizvi, P. C. Gupta, and R. K. Kaul, Planta med. [Stuttgart] 19, 222 [1971].

5 K. R. KirTikar and B. D. BAsu, Indian Medicinal Plants second Edition Vol. II, p. 854, Lalit Mohan, Allahabad 1933.

6 R. N. Chopra, S. L. Nayar, and I. C. Chopra, Glossary of Indian Medicinal Plants C.S.I.R., India 53 [1956].
2, 3 Semidiquinone - by oxidation

4, 5 semidiquinone ion

We are thankful to Dr. S. A. I. Rizvi for giving the compound. The authors S. K. G. and S. D. S. are thankful to C. S. I. R. New Delhi and Department of Atomic Energy (India) respectively for financial assistance.

7 C. P. Poole, JR.: Electron Spin Resonance, A comprehensive Treatise on Experimental Technique, International Publisher, New York 1967.

8 J. H. VAN VLECK, Phys. Rev. 74, 1168 [1948].

9 P. W. Anderson and P. R. Weiss, Rev. Mod. Phys. 25, 269 [1953]

10 A. K. Chirkov and A. A. Koкin, J. Expt. Theor. Phys. U.S.S.R. 30, 50 [1958].

11 R. Kubo and K. Tomita, Proc. Phys. Soc. Japan 9, 888 [1954].

12 B. Venkatraman and G. K. Fraenkel, J. chem. Physics 23, 588 [1955].

13 B. Venkatraman and G. K. Fraenkel, J. Amer. chem. Soc. 77, 2707 [1956]. 